\title{
Diagnostic and therapeutic recommendations for chronic pancreatitis. Recommendations of the Working Group of the Polish Society of Gastroenterology and the Polish Pancreas Club
}

\author{
Roland Kadaj-Lipka ${ }^{1}$, Michał Lipiński ${ }^{1}$, Krystian Adrych ${ }^{2}$, Marek Durlik ${ }^{3}$, Anita Gąsiorowska ${ }^{4}$, \\ Mirosław Jarosz ${ }^{5}$, Grażyna Jurkowska ${ }^{6}$, Ewa Małecka-Panas ${ }^{7}$, Grzegorz Oracz ${ }^{8}$, Mariusz Rosołowski ${ }^{6}$, \\ Barbara Skrzydło-Radomańska ${ }^{9}$, Renata Talar-Wojnarowska ${ }^{7}$, Grażyna Rydzewska ${ }^{1,10}$
}

\author{
${ }^{1}$ Clinical Department of Internal Medicine and Gastroenterology with Inflammatory Bowel Disease Unit, CSK MSWiA, Warsaw, Poland \\ ${ }^{2}$ Chair and Department of Gastroenterology and Hepatology, Medical University of Gdansk, Poland \\ ${ }^{3}$ Department of Gastroenterological Surgery and Transplantation Medicine, Central Clinical Hospital of the Ministry \\ of the Interior and Administration, Warsaw, Poland \\ ${ }^{4}$ Department of Gastroenterology, WAM-CSW University Clinical Hospital, Lodz, Poland \\ ${ }^{5}$ Department of Metabolic Diseases and Gastroenterology, Institute of Food and Nutrition, Warsaw, Department of Internal \\ Medicine and Gastroenterology, Mazovian Bródno Hospital, Poland \\ ${ }^{6}$ Department of Gastroenterology and Internal Medicine, Medical University of Białystok, Poland \\ ${ }^{7}$ Department of Gastrointestinal Diseases, Medical University of Lodz, Poland \\ ${ }^{8}$ Department of Gastroenterology, Hepatology, Nutrition Disorders and Paediatrics, Children's Memorial Health Institute, \\ Warsaw, Poland \\ ${ }^{9}$ Chair and Department of Gastroenterology with Endoscopy Laboratory, Medical University of Lublin, Poland \\ ${ }^{10}$ Department of Medicine and Health Sciences, Jan Kochanowski University, Kielce, Poland
}

Key words: chronic pancreatitis, endosonography, enzymatic replacement therapy, endoscopic treatment, surgical treatment, pancreatic cancer, guidelines, Polish Society of Gastroenterology, Polish Pancreas Club.

Address for correspondence: Department of Internal Medicine and Gastroenterology with IBD Treatment Subunit, CSK MSWiA, ul. Wołoska 137, 02-507 Warszawa, Poland

\begin{abstract}
This article describes the latest diagnostic and therapeutic recommendations in chronic pancreatitis, developed by the Working Group of the Polish Society of Gastroenterology and the Polish Pancreas Club. The recommendations refer to the diagnosis of chronic pancreatitis, autoimmune pancreatitis, conservative management, treatment of pain, and exocrine and endocrine pancreatic insufficiency, treatment of chronic pancreatitis by endoscopic and surgical methods, and oncological surveillance of chronic pancreatitis. This paper refers to the Polish recommendations published in 2011, which have been updated and supplemented. All recommendations were voted by experts of the Polish Society of Gastroenterology and the Polish Pancreas Club, who evaluated them each time on a five-degree scale, where I meant full acceptance, II - acceptance with some reservation, III - acceptance with serious reservation, IV - rejection with some reservation and V - full rejection. The results of the voting, together with a brief commentary, have been included with each recommendation put to the vote. In addition, the expert group assessed the value of clinical studies on which the statements are based, on a scale where A means high (based on meta-analyses and randomised clinical trials), B means medium (based on clinical trials and observational studies), and C means low (based mainly on expert opinion).
\end{abstract}




\section{Introduction}

Chronic pancreatitis (CP) is an inflammatory disease that causes progressive and irreversible damage to the parenchyma of the organ, leading to its atrophy and fibrosis, and the progressive development of exocrine and endocrine pancreatic insufficiency. The frequency of the $\mathrm{CP}$ in Poland has been increasing; according to some estimates, the annual incidence is 10.7 per 100,000 , the average incidence is $5-10$ per 100,000 , and the prevalence of CP in Poland has increased from 12.3 per 100,000 in 1982 to 56.3 per 100,000 in 2001 [1-3]. It is believed that the pathogenesis of the disease involves a number of confirmed as well as potential risk factors.

In most patients, the clinical picture is dominated by abdominal pain, usually paroxysmal, recurring in the form of episodes lasting less than 10 days, followed by a longer pain-free period (more often in late idiopathic (P). The symptoms may also be chronic and severe; they may be divided by 1 - to 2 -month pain-free periods (more frequently in the case of alcoholic aetiology and early idiopathic inflammation). At the advanced stage of the disease, symptoms of organ failure occur, including digestive disorders, malabsorption, and diabetes mellitus.

This paper refers to the Polish recommendations published in 2011, which have been updated and supplemented [4].

\section{Guideline development methods}

The present paper presents and discusses 40 statements concerning the terminology, diagnostics and conservative, endoscopic and surgical treatment. Basic information on the management of autoimmune pancreatitis has also been included.

Table I. A five-step scale

\begin{tabular}{lc} 
Category & Support level \\
\hline I & Full acceptance \\
\hline II & Acceptance with some reservation \\
\hline III & Acceptance with serious reservation \\
\hline V & Rejection with some reservation \\
\hline
\end{tabular}

Table II. Scale of evidence

\begin{tabular}{|c|c|}
\hline Category & Data reliability \\
\hline A & $\begin{array}{l}\text { High (based on meta-analyses and randomised } \\
\text { clinical trials) }\end{array}$ \\
\hline $\mathrm{B}$ & $\begin{array}{c}\text { Medium (based on clinical studies and } \\
\text { observational studies) }\end{array}$ \\
\hline C & Low (mainly based on expert opinion) \\
\hline
\end{tabular}

The degree of acceptance of the presented statements was assessed on the basis of the voting results of the group of experts from the Polish Society of Gastroenterology and the Polish Pancreas Club. The level of support for each statement is expressed in a five-step scale, as shown in Table I.

In addition, the group of experts assessed the value of clinical studies on which these statements are based, on the scale presented in Table II.

\section{Guidelines}

Plain X-ray of the abdominal cavity and transabdominal ultrasonography (US) may be helpful in the diagnosis of advanced CP. Assessment I - 100\% strong support, evidence level A

Plain X-ray of the abdominal cavity is an inexpensive and easily accessible method of imaging that allows the detection of focal or disseminated calcifications in the pancreas area. A limitation of this method is the fact that calcifications are only seen in $30-40 \%$ of patients with $\mathrm{CP}$, their presence indicates an advanced stage of the disease, and they may develop in the course of other pathologies, including tumours and post-traumatic lesions of this area. Abnormalities in abdominal X-ray are very rarely observed in children, therefore it is not recommended to perform this examination routinely in this age group. Transabdominal ultrasonography is a non-invasive, widely used and well tolerated method enabling the diagnosis of CP with high sensitivity (48-96\%) and specificity (75-90\%). It shows the dilated pancreatic duct (PD), calcifications (especially those measuring $>5 \mathrm{~mm}$ ) and pseudocysts [5]; however, it does not allow to identify early changes [6]. In children, transabdominal ultrasonography is the preferred diagnostic method for $\mathrm{CP}$ due to the potential harmfulness of $\mathrm{X}$-rays.

Endosonography (EUS) is the recommended method for diagnosing early changes in CP due to its highest diagnostic efficacy. Assessment I - 100\% - strong support, evidence level B

Endosonography is characterised by the highest sensitivity (85-100\%) and a very high specificity (85$100 \%$ ), second only to endoscopic retrograde cholangiopancreatography (ECPW) in the context of CP diagnosis [5]. Using the currently recommended Rosemont classification, it is possible to identify certain or probable CP, identify undefined lesions, and unequivocally rule out the disease based on a constellation of the so-called large and small criteria. Large criteria include increased 
echogenicity foci with an acoustic shade, deposits in the $\mathrm{PD}$, and "honeycomb" lobular structure. Small criteria include cysts, PD dilation above $3.5 \mathrm{~mm}$, irregular outline and hyperechogenic wall of the PD, side branches over $1 \mathrm{~mm}$ wide, hyperechogenic lesions without an acoustic shadow, and discontinuous lobular structure [7]. The method may be supplemented by elastography. Endosonography is also useful in the assessment of advanced lesions. A limitation of this method in children is the patient age and the size of diagnostic instruments.

Magnetic resonance imaging, possibly in the cholangiopancreatography option (MRI/MRCP), and computed tomography $(\mathrm{CT})$ are the recommended complementary methods in the diagnosis of CP. Typical features of CP found in abdominal MRI/ MRCP or CT allow to diagnose the disease. At the same time, the normal pancreatic image found in these scans does not allow to rule out early forms of CP. Assessment I - 100\% - strong support, evidence level $B$

Magnetic resonance cholangiopancreatography is a safe, non-invasive imaging method, which does not require exposure to iodine contrast agents or X-rays, and whose sensitivity and specificity in the diagnosis of CP are $88-91 \%$ and $92-98 \%$, respectively [5]. It shows fluid-filled structures, such as the PD, with an accuracy close to that of ECPW (92\%) [5]. On the other hand, there is no equally high correlation between the methods for visualisation of lateral branches, which in MRCP are visible only in 10-25\% of cases [8]. Magnetic resonance cholangiopancreatography enables visualisation of PD discontinuity, communication with a pseudocyst, and pancreatic fistulas to the peritoneal or pleural cavities. A significant limitation of the method is its low sensitivity in the detection of calcifications, whereas MRCP may be the only method of pancreatography in the situation of post-operative anastomoses that impair or prevent endoscopic access [9]. The use of secretin in MRCP improves the sensitivity of the examination [10, 11]. The sensitivity and specificity of spiral CT in the diagnosis of CP are $56-95 \%$ and $85-100 \%$, respectively [5]. The method allows for the detection of atrophic parenchyma, calcifications, deposits in the PD, as well as for the visualisation of intra- and peripancreatic cysts, spleen vein thrombosis, and for the evaluation of the stage of inflammatory or neoplastic pancreatic tumour. At the same time, it is not recommended to routinely perform diagnostic endoscopic retrograde cholangiopancreatography. Due to the risk of serious complications, this examination should be reserved for therapeutic procedures.

Functional tests may be useful in the diagnosis of exocrine insufficiency in $\mathrm{CP}$ in the case of ambiguous imaging results. It is recommended to measure faecal elastase-1 activity. Assessment I - 100\% strong support, evidence level B

Elastase-1 is an enzyme secreted by the pancreas, which is not broken down during the passage through the intestines and therefore its faecal level correlates well with duodenal secretion. The immunoenzymatic method, based on the use of monoclonal antibodies specific to the human body (ELISA), ensures that enzymatic substitution therapy does not change the concentration of elastase- 1 and thus does not affect the result. The test is performed using a single faecal sample; concentration above $200 \mu \mathrm{g}$ enzyme per $1 \mathrm{~g}$ of faeces is considered normal. Elastase-1 activity below $200 \mu \mathrm{g} / \mathrm{g}$ indicates mild exocrine pancreatic insufficiency, and the value below $100 \mu \mathrm{g} / \mathrm{g}$ [12], and even more below $50 \mu \mathrm{g} / \mathrm{g}$ [11], indicates severe exocrine pancreatic insufficiency. The sensitivity of the method in recognising medium severe and severe exocrine insufficiency reaches $100 \%$. Other functional tests may also be performed depending on the experience of a given institution. Faecal elastase- 1 activity measurement is of key importance due to the fact that the severity of morphological changes typical for CP found in imaging examinations does not always correlate with the degree of exocrine insufficiency. In order to rule out exocrine pancreatic insufficiency, it is recommended to determine faecal elastase- 1 in asymptomatic or mildly symptomatic patients or in case of ambiguous imaging results.

The diagnosis of autoimmune pancreatitis (AP), in the absence of consensus on diagnostic criteria, should be carried out at a centre where the full range of tests enabling differential diagnosis is available, especially in the forms with partial pancreatic involvement. Assessment I - 100\% - strong support, evidence level C

Autoimmune pancreatitis is a type of chronic inflammation that is distinguished by its specific histopathological picture and the possibility to treat with steroids. It is a form of inflammation that often occurs with symptoms of jaundice and characteristic morphological changes in the pancreas, including the presence of pathological mass $[13,14]$. Autoimmune pancreatitis 
requires very careful differential diagnosis with pancreatic cancer. There are 2 types of autoimmune pancreatitis, type 1 being a pancreatic manifestation of IgG4-dependent disease, and type 2 in which inflammatory changes are limited to the pancreas.

Type 1 is a systemic disease where changes in the pancreas occur synchronously or metachronously with involvement of other organs; the serological profile includes elevated IgG4 and the presence of IgG4 in the tissues. The peak of the disease is in the $6^{\text {th }}$ and $7^{\text {th }}$ decade of life; men are more likely to be affected ( $2: 1$ compared to women).

Type 2 - IDCP (idiopathic duct-centric pancreatitis) is characterised by infiltrations mainly of granulocytes concentrated around the ducts, periductal fibrosis leading to destruction of the ducts. The histopathological differences translate into the clinical course of each type.

Type 2 AP concerns the younger age group, there are no differences in frequency between men and women, the disease often occurs in patients with non-specific inflammatory bowel diseases.

The criteria for diagnosis are based on clinical data, imaging, serology and histopathology. The Japanese criteria include: generalised or segmental pancreatic enlargement with scarring within the PD (irregular wall) documented in US, CT, MRI; high concentration of immunoglobulins G, IgG4, and/or presence of antinuclear antibodies (ANA), rheumatoid factor (RF), or interlobular fibrosis in histolog ical examination, intensive periductal infiltration of lymphocytes and plasma cells, lymph follicles. The diagnostic criteria developed by the Mayo Clinic, HISORt, are based on imaging, serology, the presence of extrapancreatic symptoms, and response to steroid therapy $[14,15]$.

Immunoglobulins IgG4 are a common element of the diagnosis included in the Japanese, Korean and HISORt criteria. Hamano estimated the sensitivity of elevated lgG4 titre (>135 ng/dl) to $95 \%$ and the specificity to $97 \%$ in the diagnosis of AP; however, isolated elevated IgG4 level cannot be the only criterion for the diagnosis of AP.

IgG4 titre correlates with disease activity, usually decreases with treatment, and sometimes also spontaneously. Persistently elevated IgG4 concentrations during treatment may be an indicator of relapse.

Segmental pancreatic involvement requires indepth differentiation from pancreatic cancer, including thin-needle biopsy.

Treatment and monitoring of patients with AP should be carried out at specialist reference centres. Assessment I - 100\% - strong support, evidence level $C$
Indications for the treatment of AP in symptomatic patients include:

- mechanical jaundice,

- pain or involvement of other organs, including jaundice due to CBD stenosis in patients with typical morphological lesions.

In the case of asymptomatic or mildly symptomatic patients they include: finding of a pathological mass in the pancreas.

Steroids are the first line of treatment for remission induction, unless there are contraindications for their use. If steroids are ineffective or contraindicated, rituximab can be used for remission induction. Assessment I - 100\% - strong support, evidence level B

Other steroid-sparing drugs (except for rituximab), such as thiopurines, show low efficacy in remission induction when used alone. Steroids should be used at an initial dose of $0.6-1 \mathrm{mg} / \mathrm{kg} /$ day.

The recommended minimum dose is $20 \mathrm{mg}$ of prednisone. It is recommended to use the treatment for approximately 4 weeks, then reduce the dose in a typical way, usually by $5 \mathrm{mg}$ per week. Alternatively, steroid dose can be reduced from the initial dose of $40 \mathrm{mg} /$ day by $5 \mathrm{mg}$ per week to $20 \mathrm{mg} /$ day, and then reduced by $5 \mathrm{mg}$ every 2 weeks. High doses of steroids used "ex juvantibus" are not recommended.

Patients with type 1 inflammation with low disease activity at the beginning of treatment, and those with type 2 AP, do not require any maintenance treatment. Maintenance treatment should not be used routinely, although it may be necessary in selected patients with type 1 disease. Maintenance therapy is usually used for up to 3 years, with a low dose of $5 \mathrm{mg}$ of prednisone per day [16].

Endoscopic drainage of the bile ducts in case of mechanical jaundice not only prevents bile duct infection, but also allows to collect material for cytological examination to differentiate between IgG4-SC and bile duct cancer. Assessment I - 100\% - strong support, evidence level B

In the case of minor jaundice without signs of infection, steroids can be used, without the need to use bile duct stenting $[13,14]$. This is especially the case with mild jaundice without signs of infection, in which oral steroids may be sufficient treatment. 
Smoking cessation reduces pain and the risk of complications in CP. Assessment I- $100 \%$ - strong support, evidence level B

There is evidence showing the beneficial effects of smoking cessation on CP progression. It has also been observed that patients reporting chronic pain in CP are mainly smokers. However, there is no clear evidence of the relationship between pain and smoking cessation [1]. Some data indicate smoking as an independent risk factor for the development of CP and pancreatic cancer [17]. Retrospective studies have shown the relation between smoking and the earlier onset of the disease, including calcifications and diabetes mellitus $[17,18]$. In a large cohort study, the total risk of pancreatic cancer development in CP patients at 10 and 20 years after the diagnosis was $1.8 \%$ and $4 \%$, respectively [19]. In patients with hereditary $\mathrm{CP}$, smoking increases the risk of pancreatic cancer more than 50-fold [20]. At the same time, the studies indicate a positive effect of smoking cessation, resulting in the resolution or reduction of pain and the incidence of CP complications [17, 21, 22]. Absolute smoking cessation should be recommended in patients with $\mathrm{CP}$.

Alcohol cessation is recommended. Assessment I$100 \%$ - strong support, evidence level B

In patients with alcohol-induced $C P$, abstinence slows down the progression of the disease and is associated with improved pain control. The cessation of alcohol consumption in this group of patients prolongs survival and has a positive impact on the quality of life [23]. However, data on the improvement in exocrine pancreatic function resulting from alcohol abstinence are not unambiguous [23-25].

Limiting the dietary intake of fats is recommended only in case of persistent severe diarrhoea despite the appropriate enzyme replacement therapy. Assessment I - 100\% - strong support, evidence level B

There are no convincing data indicating the need to reduce dietary fats in patients with $\mathrm{CP}$; however, severe fatty diarrhoea significantly deteriorates the quality of life, causes social implications and may predispose to urolithiasis. On the other hand, restrictive reduction of fat in the diet is associated with impaired nutritional status and absorption of specific vitamins [26]. Ran- domised studies show that a diet with a fat content of at least $100 \mathrm{~g}$ fat per day is well tolerated by patients with CP provided that adequate enzyme replacement therapy is used [26]. At present, low-fat diet is not recommended in CP patients, whereas higher than normal fat intake (i.e. corresponding to approximately $30 \%$ of the daily energy requirement) may be recommended in patients with difficulties in increasing or maintaining proper body weight.

Supplementation of fat-soluble vitamins (especially vitamin $D_{3}$ ) should be considered at all levels of severity of exocrine pancreatic insufficiency in the course of CP. Assessment I - $100 \%$ - strong support, evidence level $\mathrm{C}$

In the group of patients with CP-related exocrine pancreatic insufficiency, serum levels of fat-soluble vitamins $(A, D, E, K)$ are decreased, despite the frequent lack of clinical manifestation of avitaminosis. Vitamin D deficiency precedes the occurrence of fatty diarrhoea. Additionally, CP patients are at a risk of vitamin $B_{12}$ deficiency since its metabolism is closely related to the gastrointestinal tract. In order to avoid complications (including osteopenia), the decision to start vitamin supplementation, in the absence of clinical manifestation of deficiencies, should depend on serum concentrations of specific vitamins [6].

Enzyme replacement therapy improves the quality of life in patients with CP and is the basis for the treatment of exocrine pancreatic insufficiency; however, it is not recommended for pain treatment in CP. Assessment I - 100\% - strong support, evidence level $A$

Assuming a negative feedback within the duodenal-pancreatic axis, it can be assumed that administration of exogenous pancreatic enzymes by reducing cholecystokinin secretion would lead to decreased pressure in the pancreatic ducts and relief of pain dependent on this mechanism. However, data from clinical trials, including meta-analyses evaluating this hypothesis, are divergent. Apart from one prospective multicentre study $[27,28]$, no significant benefits have been demonstrated in the analgesic effects of pancreatic enzyme products used in the form of enteric-coated capsules, which, however, may indicate insufficiently effective release of enzymes in the feedback part of the duodenum. On the contrary, studies with products without a coating that prevents inactivation in an acidic environment 
have shown a reduction in pain compared to placebo, which may be associated with additional use of drugs to reduce hydrochloric acid secretion and increased alkalinity of the duodenal environment [6]. The authors of a review of the above studies, published in 2009, do not recommend routine use of pancreatic enzyme products as a pain treatment method in the course of CP [29]. However, the practical recommendations assume the use of enzymatic therapy as a rational method of initial analgesic management, most probably acting in the mechanism of reducing a number of dyspeptic symptoms related to the beginning of digestive disorders $[6,29]$. The consequences of CP include malnutrition and fatty diarrhoea resulting from impaired digestion. Enzyme replacement therapy improves the absorption of fats and fat-soluble vitamins, reduces the severity or delays the onset of fatty diarrhoea, and improves the nutritional status of patients with CP. The time to start the therapy remains debatable.

Fatty diarrhoea remains a classic indication but occurs only in $35 \%$ of patients with exocrine insufficiency. However, asymptomatic patients with subclinical exocrine insufficiency seem to benefit from enzyme therapy. The results of a multicentre prospective clinical study published in 2003 indicate that in patients with CP the use of enzymatic substitution therapy reduces the incidence of fatty diarrhoea and pain and is associated with a significant improvement in the quality of life [28]. Taking into account the results of studies conducted so far, enzyme replacement therapy is not recommended for pain treatment in CP [1], although its application may be associated with the reduction of certain symptoms such as flatulence and gases.

Pancreatic enzymes should be used in the form of enteric capsules, containing $\mathrm{pH}$-sensitive mini-microspheres and micropellets with high lipase concentrations. The recommended dosage for the main meals is 30,000 to 40,000 lipase units. Half the dose is recommended for snacks. Assessment I $-72 \%$, II - $28 \%$ - moderate support, recommendation strength $\mathrm{A}$

The recommended dose for the main meals is 30,000-40,000 even to 50,000 lipase units per meal and can be safely increased to a maximum of 75,000 80,000 lipase units per meal $[12,30]$. Therapy can also be started with smaller doses of pancreatic enzymes.

The effectiveness of enzyme replacement therapy depends on the activity of enzymes released in the duodenum. Enteric capsules containing microspheres and micropellets sensitive to the $\mathrm{pH}$ of the environment, with a high concentration of lipase, are not inactivated in the acidic environment of the stomach thanks to the protective coating; the rapid decomposition of the coating and the release of enzymes takes place at a pH of at least 5.5. It has been shown that mini-microspheres and micropellets with a diameter of 1.0-1.2 mm release digestive enzymes simultaneously with food reaching the duodenum and are characterised by $25 \%$ higher therapeutic efficacy than microspheres and micropellets with a diameter of $1.8-2.0 \mathrm{~mm}$. At the same time, there is no clear evidence that minimicrospheres and micropellets increase the effectiveness of enzyme therapy in controlling fatty diarrhoea. The dose should be adapted to the degree of malnutrition and fat content in the meal. Randomised studies have shown that the use of 40,000 lipase units for the main meals and 20,000 units for snacks is associated with improved fat absorption, lower incidence of bowel movements and improved stool consistency [31]. The average dose of 40,000 lipase units per meal per year is associated with a significant increase in body weight, normalisation of fat absorption and the levels of retinol-binding proteins and prealbumins in most patients with CP. Oral preparations of pancreatic enzymes should be taken together with a meal or snack. If the dosing regimen involves taking more than one capsule for a meal, some benefits may be obtained by taking one capsule at the beginning of the meal and the other one in the course of the meal [1].

The recommended substitution dose in children under 4 years of age with exocrine pancreatic insufficiency is 1000 lipase units per $1 \mathrm{~kg}$ body weight per meal. In older children ( $>4$ years of age) the dose is 500 lipase units per $1 \mathrm{~kg}$ body weight per meal. The maximum daily dose should not exceed 10,000 lipase units per $1 \mathrm{~kg}$ body weight $[12,13]$.

When fatty diarrhoea cannot be controlled by enzyme replacement therapy, a proton pump inhibitor (PPI) should be additionally used. Assessment I$100 \%$ - strong support, evidence level B

In $\mathrm{CP}$ patients the $\mathrm{pH}$ in the duodenum may be lower than in healthy subjects due to decreased bicarbonate secretion, which may result in impaired release of exocrine enzymes from microspheres and micropellets. PPIs should not be used in patients with good response to enzyme replacement therapy. PPI addition is recommended in patients with persistent fatty diarrhoea despite optimal enzyme replacement therapy $[1,12]$. There are no clear data on the clinical benefit of combining PPIs and enteric-coated pancreatic enzyme products [31]. 
Non-steroidal anti-inflammatory drugs and paracetamol are recommended for the treatment of pain associated with CP. Opioid analgesics should be used as short as possible, only as temporary treatment. Assessment I - 100\% - strong support, evidence level $B$

The treatment of pain accompanying $\mathrm{CP}$ should be carried out according to the WHO (World Health Organisation) analgesic ladder. The main principle of pain management is to use the lowest possible dose and administer drugs orally [1]. Analgesics should be used when pain cannot be controlled by modifying lifestyle and diet in combination with optimal enzyme replacement therapy. Priority should be given to non-opioid agents from the group of non-steroidal anti-inflammatory drugs and paracetamol. If opioids must be used, tramadol with a relatively low potential for addiction and limited effect on the gastrointestinal tract function is recommended. If necessary, the effect of tramadol can be enhanced by the addition of tricyclic antidepressants (TCAs) or selective serotonin reuptake inhibitors (SSRIs), especially recommended in CP patients with concomitant depression [6, $19,32]$. In view of the high risk of addiction, particularly in patients with alcohol-induced CP, opioids other than tramadol should only be used in cases resistant to other forms of therapy. As an alternative, inhibitors of $\alpha 2 \delta$ subunits of calcium channels, such as gabapentin and pregabalin, may be used to control pain. Level I - paracetamol is preferred; other non-steroidal anti-inflammatory drugs (NSAIDs) are not recommended because of their adverse effect on the gastrointestinal tract. Level II - at this level of analgesia, tramadol was found to be more effective compared to morphine in patients with CP. Level III - strong opioids, including morphine.

The most important criterion for the effectiveness of enzyme therapy is the clinical improvement of the nutritional status and the resolution of gastrointestinal symptoms. Laboratory tests to evaluate fat absorption and measure the degree of exocrine pancreatic insufficiency, such as elastase 1 , can be used in patients without clinical improvement. Assessment I - $100 \%$ - strong support, evidence level B

Malnutrition secondary to exocrine pancreatic insufficiency plays a prognostic role in patients with CP. Enzyme replacement therapy aims to prevent fatty diarrhoea and weight loss; however, there is no evidence that a good clinical response clearly demonstrates the effectiveness and optimisation of the therapy. It has recently been demonstrated that an adequate clinical response to enzyme replacement therapy in patients with exocrine pancreatic insufficiency does not allow predicting proper nutritional status (assessed in the study on the basis of blood concentration of retinol-binding protein, prealbumins and transferrin, and measurement of body mass index (BMI)) [33]. Therefore, the presence or resolution of fatty diarrhoea does not appear to be an appropriate parameter to assess the efficacy of the therapy. Researchers suggest treatment with pancreatic enzyme products in patients with acute diarrhoea as well as in asymptomatic patients. It is difficult to establish recommendations for monitoring the efficiency of digestion and absorption of fats. Based on the available data, it appears that elastase 1 determination, recommended for the assessment of exocrine insufficiency, may be sufficient.

Despite the existing difficulties in optimising enzyme replacement therapy, recently published studies confirm the concept of normalisation of the nutritional parameters (both anthropometric and biochemical) as an appropriate method for evaluating the effectiveness of pancreatic enzyme therapy [34].

In the absence of improvement after higher doses of pancreatic enzymes and the addition of proton pump inhibitors to the therapy, other causes of malabsorption and dyspeptic symptoms should be considered (e.g. SIBO). Assessment I - 100\% strong support, evidence level B

Recent reports indicate a more frequent occurrence of small intestinal bacterial overgrowth (SIBO) in patients with CP [35]. This may be the reason for the lack of clinical improvement in some patients despite the use of higher doses of pancreatic enzymes and the addition of a proton pump inhibitor to the therapy. Additionally, correct interpretation is complicated by the fact that some SIBO symptoms may mimic the image of exocrine pancreatic insufficiency, and the use of PPI increases the risk of SIBO [36]. Therefore, in each case, different causes of the reported problems should be considered and appropriate treatment given, e.g. with rifaximin.

For the evaluation of endocrine pancreatic secretion, fasting blood glucose and/or $\mathrm{HbA}_{1 \mathrm{c}}$ measurements are recommended. Assessment I - 100\% strong support, evidence level A

Up to $70 \%$ of patients with CP may have impaired glucose tolerance. The likelihood of developing endocrine pancreatic insufficiency gradually increases over 
the 10 years following the diagnosis of CP. Early detection of endocrine pancreatic insufficiency is possible based on fasting blood glucose, glycated haemoglobin $\left(\mathrm{HbA}_{1 \mathrm{c}}\right)$ or oral glucose tolerance test. American and European experts recommend the determination of $\mathrm{HbA}_{1 \mathrm{c}}$ (with a cut-off point of $6.5 \%$ ), noting that fasting is not required and that individual differences are small compared to blood glucose levels [6, 37]. However, it seems that routine management for early detection of endocrine pancreatic insufficiency should include annual monitoring of fasting blood glucose.

The treatment of diabetes associated with CP does not significantly differ from the treatment of type 1 and type 2 diabetes. Assessment I-89\%, II - $11 \%$ - moderate support, evidence level C

Diabetes of a different type in the course of CP is characterised by a higher risk of hypoglycaemia and a lower tendency to ketone acidosis due to impaired glucagon secretion, malnutrition, and sometimes coexisting alcohol-induced liver failure. Complications such as nephropathy, neuropathy and retinopathy are as common as in type 1 diabetes. Dietary recommendations, with particular emphasis on improving the nutritional status, supplementing fat-soluble vitamins, and preventing hypoglycaemia, mostly coincide with those for type 1 diabetes. Patient education in the field of alcohol abstinence, balanced physical exercise and enzyme replacement therapy has an important role. Insulin therapy plays an essential role in other types of diabetes associated with CP. Despite the lack of evidence of the efficacy of oral hypoglycaemic drugs, attempts are made to use sulfonylureas, thiazolidinediones and metformin [32].

Patients with dilated main pancreatic duct without pain have no indication of endoscopic treatment. Assessment I - 100\% - strong support, evidence level B

In a theoretical model, endoscopic drainage of the PD in asymptomatic patients would be aimed at improving pancreatic juice outflow and preventing the development of pancreatic insufficiency by delaying its atrophy. However, there is a lack of convincing clinical data to support this hypothesis.

Patients without obstruction and/or dilation of the main pancreatic duct have no indication for endoscopic treatment. Assessment I - 100\% - strong support, evidence level B
In patients with frequent recurrences of pain, the best results of ECPW procedures are obtained in the case of stenoses or small deposits located in the pancreatic head. Pancreatic sphincterotomy is performed for deposit removal or stenosis dilatation and stent implantation. The efficacy of sphincterotomy alone in treating pain in the absence of obstruction or dilation of the PD is debatable.

Endoscopic treatment can be effective in a group of patients with pain problems with dilation of the main pancreatic duct secondary to lithiasis or stenosis. Assessment I - 100\% - strong support, evidence level B

Pancreatic duct obstruction may result from the presence of various lesions, which often requires the combination of endoscopic methods, such as sphincterotomy, stenosis dilatation, deposit extraction, or stenting. In a multicentre study involving a group of over 1000 patients referred for endoscopic treatment due to pain in the course of CP, in whom the obstruction of the PD was caused by the presence of deposits (18\%), stenosis (47\%), or both deposits and stenosis (32\%), the symptoms resolved in $51.4 \%$ of patients over an average period of 4.9 years [38]. Twenty years of experience with polyethylene stents in the treatment of PD stenosis have shown early efficacy in pain control between $70 \%$ and 94\%, at long-term efficacy between 52\% and 82\% [39]. A number of studies indicate that endoscopic therapy is effective in pain management, which is associated with fewer hospitalisations and reduced demand for analgesics; however, the permanence of the effect is variable [32]. An important argument for endoscopic treatment is the possibility to repeat it in case of recurrence of symptoms. Endoscopic therapy is a first-line treatment in the case of contraindications or lack of conditions for surgery, and also as temporary treatment before surgery. The factors predicting the beneficial effect of endoscopic treatment include: localisation of blocking stones in the head of the pancreas, lack of pancreatic duct stenosis, short duration of the disease and a small number of pain attacks before the use of endoscopic methods [1]. Before each endoscopic treatment, pancreatic cancer should be ruled out as a cause of the stenosis, especially in the absence of calcifications.

In selected cases, extracorporeal shock wave lithotripsy (ESWL), possibly supplemented by endoscopic therapy, may be used to treat large deposits causing obstruction of the main pancreatic duct. Assessment I - $100 \%$ - strong support, evidence level C 
The success of endoscopic treatment in the removal of calcified deposits from the PD depends on their size $(\leq 10 \mathrm{~mm})$, number $(\leq 3)$ and location (head, tail of the pancreas) [38]. Extracorporeal shock wave lithotripsy is a method of fragmentation of large deposits, which is a prerequisite for effective endoscopic unblocking of the PD. It is required in $36-68 \%$ of patients with CP, it is highly effective in fragmenting deposits (54-100\%, on average $>80 \%$ ), with pain reduction or resolution in $58-86 \%$ of patients treated with ESWL alone and $48-85 \%$ of patients additionally treated with ECPW [40]. After ESWL, only $9-30 \%$ of patients require ECPW. In the group of patients with deposits located in the head or body of the pancreas, with secondary dilation of the PD, ESWL alone reduces pain to a similar extent as in combination with ECPW, at significantly lower costs of therapy. The ESWL is the recommended method of treatment for stones in the PD. Routine supplementation with ECPW increases costs without reducing pain [41].

Pancreatic duct stenting should be continued for 6 to 12 months after pain resolution. Assessment I $88 \%, \mathrm{II}-12 \%$ - moderate support, evidence level C

When deciding on stenting in mild stenosis of the PD, the necessity of repeating ECPW with stent replacement should be taken into account; however, the time of performing the next procedure is debatable. Despite a significant probability of stent obliteration within the first quarter after implantation (65\%), its beneficial effect in pain reduction is maintained, so prophylactic stent replacement after 2-3 months is controversial [42] The preferred course of action seems to be "on-demand" replacement, based on the recurrence of symptoms associated with dilation of the PD (US, MRCP), which occurs on average after 12 months (2 to 38 months) [38, $40,43]$. Unfortunately, this procedure often requires even 4-5 ECPW procedures, stent removal is possible only in $50 \%$ of cases, and is associated with a recurrence of symptoms in $1 / 3$ of patients after 2 years. Promising results were obtained with the simultaneous implantation of several (2-4) stents removed after 6 months, which was associated with the maintenance of a good analgesic effect in $84 \%$ of patients during the average 38-month follow-up period [44]. Non-coated and partially coated self-expanding metal stents (SEMS), despite being relatively easy to implant and having a large nominal diameter, are prone to ingrowth. Although self-expanding, fully-coated stents removed after 2 months provide a significant improvement in patency, they are associated with a relatively high (up to $38 \%$ ) risk of migration [42]. Early removal of the stent should be con- sidered if there is no analgesic effect. It has not been clearly defined how long stenting of the main pancreatic duct should be continued in mild stenosis. There is no doubt that indications for surgical treatment should be considered after 6-12 months.

Early relief or resolution of pain after the procedure occurs in up to $94 \%$ of patients, while maintaining this effect for the subsequent 6 months is possible in more than $70 \%$ of cases. If the stent is maintained for 12 months, long-term analgesic effect is achieved in $2 / 3$ of patients despite the fact that stenosis subsides in only a small part of cases. Over $70 \%$ of patients in whom the stent was removed after an average of 15.7 months of implantation had not experienced recurrent pain for an average of 3.8 years [38]. The mean time of PD stenting in clinical trials was 6-12 months [27].

Endoscopic treatments are indicated as effective temporary treatment in patients with cholangitis and/or cholestasis in the course of CP. Assessment I - 88\%, II - 6\%, III - $6 \%$ - moderate support, evidence level $\mathrm{C}$

Biliary tract stenosis in the course of CP is seen in $3-23 \%$ of patients [45]. Indications for treatment include the occurrence of symptoms (jaundice, cholangitis) and/or persistent ( $>4$ weeks) asymptomatic cholestasis (serum alkaline phosphatase concentration twice the upper normal limit) [39]. The routine procedure to assess the reversibility of stenosis includes oneyear follow-up with elective replacement of the stent every 3 months. In the situation of persistent stenosis or even just a suspicion of a neoplastic process, surgical treatment is recommended, which has recently played an increasing role in the treatment of this type of diseases [1].

Observations show that implantation of a single plastic stent is associated with a recurrence of stenosis after stent removal in $62-88 \%$ of cases. Simultaneous insertion of several plastic stents instead of one is associated with much greater effectiveness in preventing recurrence of stenosis 4 years after stent removal (92\% vs. 24\%). Endoscopic stenting may be the only form of treatment for reversible stenosis caused by swelling or a pseudocyst. More often, however, irreversible stenoses due to fibrosis eventually require surgical treatment, while endoscopy plays a bridging role. SEMS placement should be considered in patients not eligible or not consenting to surgery despite a $10-62 \%$ risk of stent occlusion seen over a period of 22-50 months [27]. Promising results were obtained in the treatment of mild narrowing of the main bile duct in the course of CP with the use of 
partially coated SEMS implanted for an average period of 5 months, which was associated with the absence of recurrence of stenosis in $80 \%$ of patients in the average period of 22 months after stent removal.

Pancreatic stent replacement should be scheduled at regular intervals (e.g. every 3 months) or "on-demand" in patients with recurrent pain and pancreatic duct dilation. Assessment I - 100\% - strong support, evidence level $C$

"On-demand" stent replacement strategy is preferable as the duration of clinical improvement after insertion is unpredictable and not correlated with stent occlusion [46]. The following criteria should lead to consider discontinuation of stenting: free drainage of a contrast agent 1-2 minutes after filling the duct above the site of stenosis, easy passage of a $6 \mathrm{Fr}$ catheter through the site of stenosis, and reduction of segmental stenosis. It is worth emphasising that it is not necessary to remove the stenosis completely in order to obtain the analgesic effect $[47,48]$.

Neurolysis of the visceral plexus in pain management is not recommended due to low long-term efficacy. Assessment I-81\%, II - 19\% - moderate support, evidence level $B$

Previous versions of the guidelines for CP management developed by the Polish team (2011) analysed the blockade and neurolysis of the visceral plexus in the context of pain management. In the new version of the guidelines, we pay attention only to neurolysis of the visceral plexus. Impaired visceral plexus conduction can be achieved by blocking it (local injection of a glucocorticosteroid/anaesthetic) or by neurolysis (local injection of ethanol). Meta-analyses show that significant pain reduction can be achieved in approximately $50 \%$ of patients with CP undergoing visceral plexus blockade under EUS control [49]; however, the effect is maintained after 12 and 24 weeks only in $26 \%$ and $10 \%$ of patients, respectively. The method is also not free of complications (up to $33 \%$ ) in the form of diarrhoea, orthostatic hypotension, severe pain or pancreatic abscess.

Endoscopic drainage is recommended in symptomatic or complicated pseudocysts of the pancreas. The size of an asymptomatic cyst is not an indication for its treatment. Assessment I - 100\% strong support, evidence level A
Treatment of asymptomatic cysts and pseudocysts of the pancreas is not recommended, regardless of their size. Most of them are absorbed spontaneously about 6 weeks after the onset of acute pancreatic symptoms or CP, and only $20 \%$ of patients experience complications requiring further intervention. It is worth noting that the size of pancreatic pseudocysts does not accurately predict the probability of spontaneous absorption or development of complications [1]. Pancreatic pseudocysts are formed in the course of CP in $20-40 \%$ of patients, and spontaneously regress in $40 \%$ of cases, more often in case of lesions measuring less than $3 \mathrm{~cm}$. Symptoms (pain, severe obstruction, jaundice, weight loss) and infection of the lesion are indications for the treatment of a pseudocyst [39]. It was found that large cysts $(>6 \mathrm{~cm})$ are much more often symptomatic and therefore more often require treatment, while the large size itself of an asymptomatic and uncomplicated cyst does not constitute an indication for drainage. In a particular clinical situation, a large asymptomatic pancreatic pseudocyst may be endoscopically drained, but only after the risk of complications has been assessed by an experienced, multidisciplinary medical team, after the post-inflammatory character of the lesion has been proven, and communication with the pancreatic duct has been excluded. At reference centres, endoscopic cyst drainage becomes a treatment of choice due to lower invasiveness with similar long-term efficacy (an average of $79.6 \%$ ) as compared to surgical treatment [39]. Endoscopic drainage is recommended for the treatment of symptomatic or complicated pseudocysts due to its proven high efficacy (79.2\%) and low risk of complications (12.9\%) [45].

For the treatment of pseudocysts, endoscopic transmural drainage under endosonography control and/ or transpapillary drainage during endoscopic retrograde pancreatography are the recommended methods. Assessment I - 78\%, II - 22\% - strong support, evidence level $A$

Conventional endoscopic drainage depends on the presence of a bulge/protrusion of the gastrointestinal wall caused by the cyst, which determines the site of fistula formation. However, a significant part of cysts eligible for drainage do not cause wall protrusion, and their drainage is only possible under EUS control [50]. The effectiveness of drainage under EUS control in comparison with conventional drainage is significantly higher (100\% vs. 33\%) [51]. The EUS control significantly improves the safety of drainage owing to visualisation of the lesion (even without protrusion) and the vascular structures, which is important when collateral circulation coexists [52, 53]. Transmural drainage under EUS control is most often performed through the 
stomach wall in large $(>6 \mathrm{~cm})$ cysts well adjacent to the gastrointestinal tract. The procedure requires prior assessment of communication between the PD and the duodenum, and in case of pancreatic duct stenosis, drainage should be preceded by ECPW with dilatation. Smaller cysts (usually $<5 \mathrm{~cm}$ in diameter), which communicate with the $\mathrm{PD}$, are drained through the major duodenal papilla (transpapillary drainage) [39]. A particular complication of CP is haemorrhagic pseudocyst (pseudoaneurysm of the pancreas), which may be endoscopically drained only at experienced centres, following prior embolisation of the blood vessel supplying the lesion using interventional radiology method [54].

Percutaneous drainage of cystic pancreatic lesions is not recommended. Assessment I - $100 \%$ - strong support, evidence level C

Percutaneous drainage, with long-term efficacy in the treatment of pseudocysts estimated at approximately $50 \%$, is associated with the risk of serious complications in the form of infection of the fluid collection and/or chronic external fistula, particularly common in disconnected pancreatic tail syndrome and with concomitant PD obstruction. In addition, maintaining an external drainage system is associated with considerable discomfort for the patient. This procedure is acceptable in selected cases of large cysts as a temporary/bridge treatment. It is necessary to first differentiate between pseudocyst and cystic tumour (and to determine its type) on the basis of medical history and examination of the aspiration fluid from the cystic lesion (amylase and lipase activity, CEA concentration, microscopic examination including for the presence of mucus). In summary, percutaneous drainage of cystic lesions of the pancreas is not recommended; this management is allowed only after the absence of contact with the main pancreatic duct has been documented and the nature of the lesion has been determined (differentiation between pseudocyst and cystic tumour).

Qualification for surgery should be carried out by a team of surgeons from a highly specialised centre; the decision should take into account the possible most sparing operation. Assessment I - 100\% - strong support, evidence level C

The currently recommended drainage operations and pancreatic parenchyma-saving surgeries are asso- ciated with low mortality and good pain control in the early stage [55].

The indications for surgical treatment include severe pain (not responding to conservative treatment), suspicion of pancreatic cancer, complications of $\mathrm{CP}$, and failure of endoscopic treatment. Assessment I - 88\%, II - 12\% - moderate support, evidence level $B$

Studies following the natural course of CP indicate that a significant number of patients (47-80\%) experience spontaneous pain relief with the duration of the disease [56, 57]; however, due to unpredictability of this phenomenon, methods to alleviate pain must be sought. There is no specific level of pain or period of time after which surgical treatment should be undertaken; however, delaying the decision results in deterioration of the quality of life, increased medical and social costs [58], and dependence on opioid analgesics. There are no prospective randomised studies comparing conservative and surgical treatment of pain; however, there are indications as to the effectiveness of surgical treatment [59-63]. Moreover, two randomised studies demonstrated the superiority of surgical treatment over endoscopic treatment in pain control in CP patients with pancreatic duct stenosis $[59,60,64]$. The suspicion of pancreatic cancer in $\mathrm{CP}$ always implies resection surgery. Cystic tumours of the pancreas (e.g. IPMN) should be considered in order to avoid conservative treatment, which is not correct in this case; acute pancreatitis should also be taken into account in order to avoid unnecessary resection. Delay in the treatment of pancreatic cancer due to misdiagnosis is usually associated with fatal outcomes $[65,66]$. Surgical drainage of the bile ducts is recommended in the case of chronic jaundice (lasting more than 1 month), acute cholangitis, and secondary biliary tract stones. Common bile duct stenosis can be effectively treated with hepatojejunostomy [67]. Duodenal stenosis affects less than $1 \%$ of patients and, if not associated with other complications of CP, can be treated by gastrojejunostomy [67]. Symptomatic pancreatic pseudocysts may be treated endoscopically and surgically [68] by creating anastomosis with the intestine on the Roux-en-Y loop or with the stomach, or by drainage operations with the coexistence of pancreatic duct dilation [69]. In order to optimise the results of pain management, reduce the risk of exocrine pancreatic insufficiency, and improve QoL, it is recommended to consider surgical treatment early [1]. 
The choice of the procedure type depends on the degree of pancreatic duct dilation and the presence and location of tumour. Assessment I - 100\% - strong support, evidence level B

Surgical drainage should be performed when the main pancreatic duct is dilated to at least $5 \mathrm{~mm}$, once a growth process has been ruled out; the length of the anastomosis has a significant impact on the late results of surgery [70-73]. It is associated with good pain control (80\%) and saving the endo- and exocrine function of the gland [74]. Drainage operation combined with partial pancreatic head resection is indicated in patients with inflammatory tumour mass in the head of the pancreas [75]. Pancreatoduodenectomy is associated with good results in the treatment of $C P$ [74, 76-80]. Despite the recommendation for drainage and mixed surgeries, it remains the most widely used surgical intervention [81], and is the standard procedure for suspected pancreatic head cancer. Distal resection is useful for suspicion of pancreatic cancer located in the tail and pseudocyst with simultaneous bleeding and impaired pancreatic juice outflow towards the duodenum.

Surgical treatment of pseudocysts should be reserved for cases where there are no conditions for endoscopic drainage and/or other complications requiring surgery coexist. Assessment I - 100\% strong support, evidence level C

Endoscopic treatment is recommended for uncomplicated cases where cysts are within reach of the endoscope. Otherwise, surgical treatment is preferred [1]. Surgical internal drainage consisting in the formation of a fistula between the pseudocyst cavity and the lumen of the stomach or the intestine is associated with higher invasiveness with similar effectiveness and risk of complications as compared to endoscopic drainage [53]. Relative contraindications to drainage are the distance between the cyst wall from the stomach or duodenal wall exceeding $1 \mathrm{~cm}$ and the presence of varicose veins and/or large blood vessels between the aforementioned structures [54, 82]. The operation should be performed when repeated endoscopic drainage remains ineffective. Surgical treatment may be preferred in cases of coexistence of bile duct and/or duodenal stenosis in the course of CP as well as in cases of significant dilation of the PD, regardless of the presence of inflammatory tumour in the head of the pancreas [6].
Patients with hereditary pancreatitis and a positive family history of pancreatic cancer (at least 2 family members with the disease) should be tested for pancreatic adenocarcinoma starting from the age of 35. Annual EUS scans and serum CA 19-9 tests are recommended. Assessment I $-82 \%$, II $-12 \%$, III - $6 \%$ - moderate support, evidence level B

Epidemiological data indicate that the risk of developing pancreatic cancer is 50 to 70 times higher in patients with hereditary CP. Age-related risk increases significantly between the ages of 40 and 50, reaching $54 \%$ at the age of 70 . All patients with a phenotype and/ or genotype specific to hereditary CP should be advised to refrain from smoking or drinking alcohol. In this group of patients, annual screening for pancreatic cancer is recommended, with examinations such as EUS, multi-slice spiral CT, or MRI/MRCP [83]. The choice of the method depends on the availability and preferences of the centre. We recommend annual tests for pancreatic cancer study including EUS and determination of CA 19-9 marker. All patients with a positive history of hereditary pancreatitis should have genetic tests performed.

In the rest of the population, routine examinations for pancreatic cancer are not currently recommended; however, in each case, the appearance of new worrying symptoms requires detailed diagnosis. Assessment I - 88\%, II - 12\% - moderate support, evidence level $\mathrm{C}$

Due to the prevalence of pancreatic cancer and the limited sensitivity and specificity of screening, routine population tests are not recommended. The identification of risk groups where screening could be cost-effective is currently the subject of intensive studies. It seems that newly detected diabetes mellitus over 50 years of age, until about 36 months after its detection, may constitute a risk group for screening purposes; however, to date, literature data on the cost-effectiveness of tests in this group of patients are not sufficient to recommend such screening at this time. Routine screening is also not recommended in the group of patients with CP, despite the known increased relative risk of cancer in this population [84].

Determining the aetiology of CP allows to predict the course of the disease and determine the risk of complications. Assessment I - 75\%, II - 25\% moderate support, evidence level $B$ 
The course of the disease and the risk of pancreatic cancer significantly differ depending on the aetiology of CP $[85,86]$. Epidemiological studies have shown that calcifications and exocrine and endocrine insufficiency occur after a shorter duration of the disease in case of alcohol aetiology compared to other causes. Alcohol cessation may reduce the rate of progression, reduce $\mathrm{CP}$-related pain, and partially restore the exocrine function of the pancreas [23]. Tobacco smoking has been recognised as an independent risk factor for the development of CP $[17,87]$. Patients with early onset of $C P$, in particular with a genetic background ( $<20$ years), are at a significantly higher risk of developing pancreatic cancer; however, smoking cessation may reduce the risk in this group. Patients with different genetic mutations have different clinical presentations (e.g. later development of diabetes) $[85,86]$.

Genetic tests should be recommended in patients with a positive family history of pancreatitis or early disease onset (<20 years). Assessment I - 100\% - strong support, evidence level C

Genetic testing is not recommended in all patients with CP. It is worth noting that the results of genetic tests do not affect the management; however, they may help the patient to better understand the nature of the disease, and may also have an impact on the family plans of the patient. Genetic studies are not recommended in patients with alcohol-induced CP, although various mutations associated with a higher risk of developing this form of the disease are known [1].

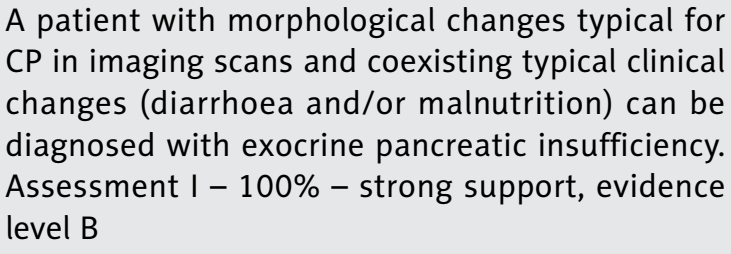

The finding of typical morphological changes characteristic of CP with coexistence of symptoms (e.g. diarrhoea, malnutrition, flatulence, abdominal pain and dyspepsia) allows to diagnose exocrine pancreatic insufficiency and start enzyme replacement therapy. This situation does not require prior evaluation of the exocrine function of the pancreas, but only verification of clinical improvement 4-6 weeks after the start of therapy [1].

\section{References}

1. Löhr JM, Dominguez-Munoz E, Rosendahl J, et al. United European Gastroenterology evidence-based guidelines for the diagnosis and therapy of chronic pancreatitis (HaPanEU). Unit Eur Gastroenterol J 2017; 5: 153-99.

2. Jarosz M, Dzieniszewski J, Orzeszko M. 20-letnie, prospektywne obserwacje epidemiologiczno-kliniczne przewlekłego zapalenia trzustki. Gastroenterol Pol 2003; 10: 371-8.

3. Dzieniszewski J. Przewlekłe zapalenie trzustki, uwagi diagnostyczne i terapeutyczne. Gastroenterologia w Codziennej Praktyce Lekarskiej 2004; 7: 3-13.

4. Żuk K, Czkwianianc E, Degowska M, et al. Zalecenia diagnostyczne i terapeutyczne w przewlekłym zapaleniu trzustki. Rekomendacje Grupy Roboczej Konsultanta Krajowego w dziedzinie Gastroenterologii i Polskiego Klubu Trzustkowego. Prz Gastroenterol 2011; 6: 339-52.

5. Fry LC, Mönkemüller K, Malfertheiner P. Diagnosis of chronic pancreatitis. Am J Surg 2007; 94 (Suppl): S45-52.

6. Bornman PC, Botha JF, Ramos JM. Guideline for the diagnosis and treatment of chronic pancreatitis. S Afr Med J 2010; 100: 845-60.

7. Catalano MF, Sahai A, Levy M, et al. EUS-based criteria for the diagnosis of chronic pancreatitis: the Rosemont classification. Gastrointest Endosc 2009; 69: 1262-3.

8. Yamaguchi K, Chijiwa K, Shimizu S, et al. Comparison of endoscopic retrograde and magnetic resonance cholangiopancreatography in the surgical diagnosis of pancreatic disease. Am J Surg 1998; 175: 203-8.

9. Mönkemüller KE, Kahl S, Malfertheiner P. Endoscopic therapy of chronic pancreatitis. Dig Dis 2004; 22: 280-91.

10. Hellerhoff KJ, Helmberger $\mathrm{H} 3^{\text {rd }}$, Rosch T, et al. Dynamic MR pancreatography after secretin administration: image quality and diagnostic accuracy. AJR Am J Roentgenol 2002; 179: 121-9.

11. Manfredi R, Lucidi V, Gui B, et al. Idiopathic chronic pancreatitis in children: MR cholangiopancreatography after secretin administration. Radiology 2002; 224: 675-82.

12. Biankin A, Oliver $M$, Pearce $C$, et al. Australasian treament guidelines for the management of pancreatic exocrine insufficiency. March 2010.

13. Párniczky A, Abu-El-Haija M, Husain S, et al. EPC/HPSG evidence-based guidelines for the management of pediatric pancreatitis. Pancreatology 2018; 18: 146-60.

14. Shimosegawa T, Chari ST, Frulloni L, et al. International consensus diagnostic criteria for autoimmune pancreatitis: guidelines of the International Association of Pancreatology. Pancreas 2011; 40: 352e8.

15. Umehara H, Okazaki K, Masaki Y, et al. Comprehensive diagnostic criteria for IgG4-related disease (IgG4-RD), 2011. Mod Rheumatol 2012; 22: 21-30.

16. Masamune A, Nishimori I, Kikuta K, et al. Randomised controlled trial of long-term maintenance cortocisteroid therapy in patients with autoimmune pancreatitis. Gut 2017; 66: 487-494.

17. Maisonneuve P, Lowenfels AB, Mullhaupt B, et al. Cigarette smoking accelerates progression of alcoholic chronic pancreatitis. Gut 2005; 54: 510-4.

18. Lowenfels, Maisonneuve P, Cavallini G, et al. Cigarette smoking as a risk factor for pancreatic cancer in patient with hereditary pancreatitis. JAMA 2001; 286: 169-70. 
19. Maisonneuve P, Frulloni L, Mullhaupt B, et al. Impact of smoking on patients with idiopathic chronic pancreatitis. Pancreas 2006; 33: 163-8.

20. Lowenfels AB, Maisonneuve P, Cavallini G, et al. Pancreatitis and the risk of pancreatic cancer. International Pancreatitis Study Group. N Engl J Med 1993; 328: 1433-7.

21. Lowenfels AB, Maisonneuve P, Dimagno EP, et al. Hereditary pancreatitis and the risk of pancreatic cancer. International Hereditary Pancreatitis Study Group. J Natl Cancer Inst 1997 89: 442-6.

22. Imoto M, Di Magno EP. Cigarette smoking increases the risk of pancreatic calcification in late-onset but not early-onset idiopathic chronic pancreatitis. Pancreas 2000; 21: 115-9.

23. Schneider A, Pfutzer RH. Treatment of alcoholic pancreatitis. Dig Dis Sci 2005; 23: 241-6.

24. Chauhan S, Forsmark CE. Pain management in chronic pancreatitis: a treatment algorithm. Best Pract Res Clin Gastroenterol 2010; 24: 323-35.

25. Gullo L, Barbara L, Labo G. Effect of cessation of alcohol use on the course of pancreatic dysfunction in alcoholic pancreatitis. Gastroenterology 1988; 95: 1063-8.

26. Safdi M, Bekal K, Martin S, et al. The effects of oral pancreatic enzymes (Creon 10 capsule) on steatorrhea a multicenter placebo-controlled, parallel group trialin subjects with chronic pancreatitis. Pancreas 2006; 33: 156-62.

27. Tringali A, Boskoski I, Costamagna G. The role of endoscopy in the therapy of chronic pancreatitis. Best Pract Res Clin Gastroenterol 2008; 22: 145-65.

28. Czakó L, Takács T, Hegyi P, et al. Quality of life assessment after pancreatic enzyme replacement therapy in chronic pancreatitis. Can J Gastroenterol 2003; 17: 597-603.

29. Winstead NS, Wilcox CM. Clinical trials of pancreatic enzyme replacement for painful chronic pancreatitis a review. Pancreatology 2009; 9: 344-50.

30. Domínguez-Munoz JE. Pancreatic enzyme therapy for pancreatic exocrine insufficiency. Curr Gastroenterol Rep 2007; 9 : 116-22.

31. Nakamura T, Arai Y, Tando Y, et al. Effect of omeprazole on changes in gastric and upper small intestine $\mathrm{pH}$ levels in patients with chronic pancreatitis. Clin Ther 1995; 17: 448-59.

32. Frulloni L, Falconi M, Gabbrielli A, et al. Italian consensus guidelines for chronic pancreatitis. Dig Liver Dis 2010; 42 Suppl. 6: S381-406.

33. Domínguez-Munoz JE, Iglesias-García J. Oral pancreatic enzyme substitution therapy in chronic pancreatitis: is clinical response an appropriate marker for evaluation of therapeutic efficacy? JOP J Pancreas (Online) 2010; 11: 158-62.

34. Lindkvist B, Phillips ME, Dominguez-Munoz JE. Clinical, anthropometric and laboratory nutritional markers of pancreatic exocrine insufficiency: prevalence and diagnostic use. Pancreatology 2015; 15: 589-97.

35. Capurso G, Signoretti M, Archibugi L, et al. Systematic review and meta-analysis: small intestinal bacterial overgrowth in chronic pancreatitis. Unit Eur Gastroenterol J 2016; 4: 697-705.

36. Ní Chonchubhair HM, Bashir Y, Dobson M, et al. The prevalence of small intestinal bacterial overgrowth in non-surgical patients with chronic pancreatitis and pancreatic exocrine insufficiency (PEI). Pancreatology 2018; 18: 379-85.
37. International Expert Committee. International Expert Committee report on the role of the A1c assay in the diagnosis of diabetes. Diabetes Care 2009; 32: 1327-34.

38. Rosch T, Daniel S, Scholz M, et al. Endoscopic treatment of chronic pancreatitis: a multicenter study of 1000 patients with long-term follow-up. Endoscopy 2002; 34: 765-71.

39. Nguyen-Tang T, Dumonceau JM. Endoscopic treatment in chronic pancreatitis, timing, duration and type of intervention. Best Pract Res Clin Gastroenterol 2010; 24: 281-98.

40. Delhaye M, Matos C, Deviere J. Endoscopic technique for the management of pancreatitis and its complications. Best Pract Res Clin Gastroenterol 2004; 18: 155-81.

41. Dumonceau JM, Costamagna G, Tringali A, et al. Treatment for painful calcified chronic pancreatitis: extracorporeal shock wave lithotripsy versus endoscopic treatment: a randomised controlled trial. Gut 2007; 56: 545-52.

42. Parkdo H, Kim MH, Moon SH, et al. Feasibility and safety of placement of a newly designed, fully covered self expandable metals stent for refractory benign pancreatic ductal strictures: a pilot study (with video). Gastrointest Endosc 2008; 68: 1182-9.

43. Eleftherladis N, Dinu F, Delhaye $M$, et al. Long-term outcome after pancreatic stenting in severe chronic pancreatitis. Endoscopy 2005; 37: 223-30.

44. Costamagna G, Bulajic M, Dumonceau JM, et al. Multiple stenting of refractory pancreatic duct strictures in severe chronic pancreatitis: long-term results. Endoscopy 2006; 38: 254-9.

45. Lerch MM, Stier A, Wahnschaffe U, Mayerle J. Pancreatic pseudocysts: observation, endoscopic drainage, or resection? Dtsch Artztebl Int 2009; 106: 614-21.

46. Morgan DE, Smith JK, Hawkins K, et al. Endoscopic stent therapy in advanced chronic pancreatitis: relationships between ductal changes, clinical response, and stent patency. Am J Gastroenterol 2003; 98: 821-6.

47. Smits ME, Badiga SM, Rauws EA, et al. Long-term results of pancreatic stents in chronic pancreatitis. Gastrointest Endosc 1995; 42: 461-7.

48. Ponchon T, Bory RM, Hedelius F, et al. Endoscopic stenting for pain relief in chronic pancreatitis: results of a standardized protocol. Gastrointest Endosc 1995; 42: 452-6.

49. Kaufman M, Singh G, Das S, et al. Efficacy of endoscopic ultrasound-guided celiac plexus block and celiac plexus neurolysis for managing abdominal pain associated with chronic pancreatitis and pancreatic cancer. J Clin Gastroenterol 2010; 44: 127-34.

50. Park DH, Lee SS, Moon SH, et al. Endoscopic ultrasound-guided versus conventional transmural drainage for pancreatic pseudocysts: a prospective randomized trial. Endoscopy 2009; 41: 842-8.

51. Varadarajulu S, Christein JD, Tamhane A, et al. Prospective randomized trial comparing EUS and EGD for transmural drainage of pancreatic pseudocysts (with videos). Gastrointest Endosc 2008; 68: 1102-11.

52. Kahaleh M, Shami VM, Conaway MR, et al. Endoscopic ultrasound drainage of pancreatic pseudocyst: a prospective comparison with conventional endoscopic drainage. Endoscopy 2006; 38: 355-9.

53. Lopes CV, Pesenti C, Bories E, et al. Endoscopic-ultrasound guided endoscopic transmural drainage of pancreatic pseudocysts and abscesses. Scand J Gastroenterol 2007; 42: 524-9. 
54. Udd M, Leppäniemi AK, Bidel S, et al. Treatment of bleeding pseudoaneurysms in patients with chronic pancreatitis. World J Surg 2007; 31: 504-10.

55. Arslanlar S, Jain R. Benign biliary strictures related to chronic pancreatitis: balloons, stents or surgery. Curr Treat Options Gastroenerol 2007; 10: 369-75.

56. Ammann RW, Muellhaupt B. The natural history of pain in alcoholic chronic pancreatits. Gastroenterology 1999; 116 1132-40.

57. Lankisch PG, Seidensticker F, Lohr-Happe A, et al. The course of pain is the same in alcohol- and nonalcohol-inducted chronic pancreatitis. Pancreas 1995; 10: 338-41.

58. Howard TJ, Jones JW, Sherman S, et al. Impact of pancreatic head resection on direct medical costs in patients with chronic pancreatitis. Ann Surg 2001; 234: 661-7.

59. Cahen DL, Gouma DJ, Nio Y, et al. Endoscopic versus surgical drainage of the pancreatic duct in chronic pancreatitis. N Engl J Med 2007; 356: 676-84.

60. Dite P, Rozuicka M, Zboril V, et al. A prospective, randomized trial comparing endoscopic and surgical therapy for chronic pancreatitis. Endoscopy 2003; 35: 553-8.

61. Farkas G, Leindler L, Daroczi $M$, et al. Prospective randomized comparison of organ-preserving pancreatic head resection with pylorus-preserving pancreaticoduodenectomy. Langenbecks Arch Surg 2006; 391: 338-42.

62. Izbicki JR, Bloechle C, Broering DC, et al. Extended drainage versus resection in surgery for chronic pancreatitis: a prospective randomized trial comparing the longitudinal pancreaticojejunostomy combine with local pancreatic head excision with the pylorus-preserving pancreaticoduodenectomy. Ann Surg 1998; 228: 771-9.

63. Koninger J, Seiler CM, Sauerland S, et al. Duodenum-preserving pancreatic head resection - a randomized controlled trial comparing the original Beger procedure with Berne modification. Surgery 2008; 143: 490-8.

64. Cahen D, Gouma DJ, Laramee P, et al. Long-term outcomes of endoscopic vs surgical drainage of the pancreatic duct in patients with chronic pancreatitis. Gastroenterology 2011; 141: 1690-5.

65. Sakorafas GH, Sarr MG. Pancreatic cancer after surgery for chronic pancreatitis. Dig Liv Dis 2003; 35: 482-5.

66. Van Gulik TM, Moojen TM, van Geenen R, et al. Differential diagnosis of focal pancreatitis and pancreatic cancer. Ann Onco 1999; 10 Suppl. 4: 85-8.

67. Vijungco JD, Prinz RA. Management of biliary and duodenal complications of chronic pancreatitis. Worl J Surg 2003; 27 1258-70.

68. Aghdassi A, Mayerle J, Kraft M, et al. Diagnosis and treatment of pancreatic pseudocysts in chronic pancreatitis. Pancreas 2008; 36: 105-12.

69. Nealon WH, Walser E. Duct drainage alone is sufficient in the operative management of pancreatic pseudocyst in patients with chronic pancreatitis. Ann Surg 2003; 237: 614-20.

70. Adams DB, Ford MC, Anderson MC, et al. Outcome after lateral pancreaticojejunostomy for chronic pancreatitis. Ann Surg 1994; 219: 481-7.

71. Greenlee HB, Prinz RA, Aranha GV. Long-term results of sideto-side pancreaticojejunostomy. World J Surg 1990; 14: 70-6.
72. Markowitz JS, Rattner DW, Warshaw AL. Failure of symptomatic relief after pancreaticojejunal decompression for chronic pancreatitis. Strategies of salvage. Arch Surg 1994; 129: 374-9.

73. Prinz RA, Aranha GV, Greenlee HB. Redrainage of the pancreatic duct in chronic pancreatitis. Am J Surg 1986; 151: 150-6.

74. Sakorafas GH, Zobolas B. Lateral pancreatojejunostomy in the surgical management of chronic pancreatitis. Current concepts and future perspectives. Dig Liv Dis 2001; 33: 187-91.

75. Diener MK, Rahbari NN, Fischer L. Duodenum-preserving pancreatic head resection versus pancreatoduodenectomy for surgical treatment of chronic pancreatitis. Ann Surg 2008; 247: 950-61.

76. Jimenez RE, Fernandez-del Castillo C, Rattner DW, et al. Outcome of pancreaticoduodenectomy with pylorus preservation or with antrectomy in the treatment of chronic pancreatitis. Ann Surg 2000; 231: 293-300.

77. Jimenez RE, Fernandez-del Castillo C, Rattner DW, et al. Pylorus preserving pancreaticoduodenectomy in the treatment of chronic pancreatitis. World J Surg 2003; 27: 1211-6.

78. Sakorafas GH, Farnell MB, Nagorney DM, et al. Pancreatoduodenectomy for chronic pancreatitis: long-term results in 105 patients. Arch Surg 2000; 135: 517-23.

79. Schnelldorfer T, Lewin DN, Adams DB. Operative management of chronic pancreatits: long-term results in 372 patients. J Am Coll Surg 2007; 204: 1039-45.

80. Witzigmann H, Max D, Uhlmann D, et al. Outcome after duodenum-preserving pancreatic head resection is improved compared with classic Whipple procedure in the treatment of chronic pancreatitis. Surgery 2003; 134: 53-62.

81. Varghese TK, Bell RH Jr. Duodenum-preserving head resection for chronic pancreatitis: an institutional experience and national survey of usage. Surgery 2007; 142: 588-93.

82. Johnson MD, Walsh RM, Henderson JM, et al. Surgical versus nonsurgical management of pancreatic pseudocysts. J Clin Gastroenterol 2009; 43: 586-90.

83. Weckman L, Kylanpaa ML, Puolakkainen P, Halttunen J. Endoscopic treatment of pancreatic pseudocysts. Surg Endosc 2006; 20: 603-7.

84. Deviere J, Bueso $\mathrm{H}$, Baize $\mathrm{M}$, et al. Complete disruption of the main pancreatic duct: endoscopic management. Gastrointest Endosc 1995; 42: 445-51.

85. Howes N, Lerch MM, Greenhalf W, et al. Clinical and genetic characteristics of hereditary pancreatitis in Europe. Clin Gastroenterol Hepatol 2004; 2: 252-61.

86. Keim V, Bauer N, Teich N, et al. Clinical characterization of patients with hereditary pancreatitis and mutations in the cationic trypsinogen gene. Am J Med 2001; 111: 622-6.

87. Talamini G, Bassi C, Falconi $M$, et al. Pain relapses in the first 10 years of chronic pancreatitis. Am J Surg 1996; 171: 565-9.

Received: 17.08 .2018

Accepted: 17.08 .2018 